\title{
Retraction: Fast and long-range triplet exciton diffusion in metal-organic frameworks for photon upconversion at ultralow excitation power
}

\author{
Prasenjit Mahato, Angelo Monguzzi, Nobuhiro Yanai, Teppei Yamada and Nobuo Kimizuka
}

Nature Materials 14, 924-930 (2015); published online 3 August 2015; retracted after print 24 November 2016.

We wish to retract this Article due to concerns with some data related to upconversion in the solid-state samples presented in Fig. 4d,e, and to the reproducibility check of the triplet diffusion constant provided in the Supplementary Information.

In this Article, we reported fast triplet energy migration and efficient photon upconversion at low excitation intensity in metal-organic frameworks (MOFs). We have since been able to observe the upconverted emission from MOFs both in benzene dispersions and in polymeric films; hence, the concept of photon upconversion in MOFs based on triplet energy migration remains valid. However, we are now unable to observe solid-state upconversion emission at the low excitation intensity reported in Fig. $4 \mathrm{~d}$,e, and to quantitatively reproduce the triplet diffusion constants in MOFs reported in Supplementary Figs 8-13 and Supplementary Tables 1-3. Since these are key parameters of this paper, all authors wish to retract this Article. We deeply regret these circumstances and sincerely apologize to the scientific community for the inconvenience this publication has caused to others.

\section{Retraction: Framing upconversion materials}

Yoan C. Simon and Christoph Weder

Nature Materials 14, 864-865 (2015); published online 3 August 2015; retracted after print 24 November 2016.

In view of the fact that the Article by P. Mahato et al. (Nature Materials 14, 924-930; 2015) has been retracted by the authors, we wish to retract this News \& Views highlighting the study as it relied on the validity of the data presented therein. 